\title{
Properties of the characteristic polynomials and spectrum of Pn and Cn
}

\author{
Essam El Seidy ${ }^{1}$, Salah Eldin Hussein ${ }^{2}$, AtefAboelkher ${ }^{3 *}$ \\ ${ }^{I}$ Department of Mathematics, Faculty of Science, Ain Shams University, Abbassia, Cairo, Egypt \\ ${ }^{2}$ Department of Mathematics, Faculty of Science, Assiut University, Assiut, Egypt \\ *Corresponding authorE-mail:atefmohamed55@yahoo.com
}

\begin{abstract}
We consider a finite undirected and connected simple graph $G(E, V)$ with vertex set $V(G)$ and edge set $E(G)$. We calculated the general formulas of the spectra of a cycle graph and path graph. In this discussion we are interested in the adjacency matrix, Laplacian matrix, signless Laplacian matrix, normalized Laplacian matrix, and seidel adjacency matrix.
\end{abstract}

Keywords: Laplacian Matrix; Signless Laplacian Matrix; Normalized Laplacian Matrix; Seidel Adjacency Matrix; Spectral.

\section{Introduction}

Graph theory and its applications has a long history, in structural mechanics and in particular Nodal ordering and graph partitioning are well documented in the literature, Kaveh [12-13]. Algebraic graph theory can be considered as a branch of graph theory, where eigenvalues and eigenvectors of certain matrices are employed to deduce the principal properties of a graph. In fact eigenvalues are closely related to most of the invariants of a graph, linking one external property to another. These eigenvalues play a central role in our fundamental understanding of graphs. Most of the definitions on algebraic graph theory in the present interesting books such as Biggs [2], Cvetković et al. [5], and Godsil and Royle [11]. One of the major contributions in algebraic graph theory is due to Fiedler [10], where the properties of the second eigenvalue and eigenvector of the Laplacian of a graph have been introduced This eigenvector, known as the Fiedler vector is used in graph nodal ordering and bipartition, Refs. [15-18].

The Laplacian matrix of a graph and its eigenvalues can be used in several areas of mathematical research and have a physical interpretation in various physical and chemical theories. The related matrix - the adjacency matrix of a graph and its eigenvalues were much more investigated in the past than the Laplacian matrix. In the same time, the Laplacian spectrum is much more natural and more important than the adjacency matrix spectrum because of it numerous application in mathematical physics, chemistry and financial mathematics (see papers $[1,3,4,6,7,9]$ ).

Throughout this paper it is assumed that all graphs are undirected and simple connected [without loops or multiple edges] and all matrices are real and symmetric.

The following are the five different types of matrices that will take care for a graph $G$ with $n$ vertices $v_{i} ; i=1,2, \ldots n$ :

The adjacency matrix, $A=A(G)=a_{i j}$ of $G$ is an $n \times n$ symmetric matrix,

$$
\mathrm{a}_{\mathrm{ij}}= \begin{cases}1 & \text { if } \mathrm{v}_{\mathrm{i}} \mathrm{v}_{\mathrm{j}} \in \mathrm{E}, \\ 0 & \text { otherwise. }\end{cases}
$$

The Laplacian matrix ofG is the matrix $L=L(G)=l_{i j}=D-A$,

$$
l_{i j}=\left\{\begin{array}{cc}
d_{i} & \text { if } i=j \\
-1 & \text { if } i j \in E \\
0 & \text { otherwise }
\end{array}\right.
$$

Where $D$ is a diagonal matrix $\left(D=\left(d_{1}, d_{2}, \ldots, d_{n}\right)\right)$.

The signless Laplacian matrix of $\mathrm{G}$ is the matrix $\mathrm{Q}=\mathrm{Q}(\mathrm{G})=\mathrm{q}_{\mathrm{ij}}=\mathrm{D}+\mathrm{A}$,

$$
q_{i j}=\left\{\begin{array}{cc}
d_{i} \quad \text { if } i=j, \\
1 \quad \text { if } i j \in E, \\
0 \text { otherwise }
\end{array}\right.
$$

The normalized Laplacian matrix of $G$ is the matrix $£=£(G)=€_{\mathrm{ij}}$,

$$
E_{i j}=\left\{\begin{array}{cc}
1 & \text { if } i=j, \\
-\frac{1}{\sqrt{d_{i} d_{j}}} & \text { if } i j \in E \\
0 & \text { otherwise }
\end{array}\right.
$$

The Seidel adjacency matrix of a graph $\mathrm{G}$ with adjacency matrix A is the matrix $S$ defined by

$\mathrm{S}=\mathrm{J}-\mathrm{I}-2 \mathrm{~A}=\mathrm{s}_{\mathrm{ij}}$ (Where $\mathrm{J}$ is the square matrix with all entries are equal one), i.e.

$$
s_{i j}=\left\{\begin{array}{cc}
0 & \text { if } i=j, \\
-1 & \text { if } i j \in E, \\
1 & \text { if } i j \notin E .
\end{array}\right.
$$

Definition 1.1: A path graph $P_{n}$ is a connected graph of $n$ vertices where 2 vertices are pendant and the other $n-2$ vertices are of degree 2. A path has $n-1$ edges.

Definition 1.2: A cycle graph $C_{n}$ is a connected graph on $n$ vertices where all vertices are of degree 2. A cycle graphcan be created from a path graph by connecting the two pendant vertices in the path by an edge. A cycle has an equal number of vertices and edges. 
Definition 1.3: The characteristic polynomial of a square matrix A as follows:

$$
\varphi(A, \lambda)=\operatorname{det}(\lambda I-A), \operatorname{or} \varphi(A, \lambda)=\operatorname{det}(A-\lambda I),
$$

Where I is the identity matrix and $\lambda$ is the eigenvalues of $A$.

Definition 1.4: The roots of $\varphi(G, \lambda)$ are the eigenvalues of $A(G)$ We will call them also the eigenvalues of $G$.

Definition 1.5: The spectrum of a finite graph $G$ is the set of eigenvalues of the adjacency matrix $A(G)$ to gether with their multiplicities.

Suppose A (G) has s distinct eigenvalues $\lambda_{1}>\lambda_{2}>\cdots>\lambda_{s}$, with multiplicities $m\left(\lambda_{1}\right), m\left(\lambda_{2}\right), \ldots, m\left(\lambda_{s}\right)$ respectively, then we shall write

$$
\operatorname{Spec}(G)=\left(\begin{array}{cccc}
\lambda_{1} & \lambda_{2} & \cdots & \lambda_{s} \\
m\left(\lambda_{1}\right) & m\left(\lambda_{2}\right) & \cdots & m\left(\lambda_{s}\right)
\end{array}\right)
$$

Or

$$
\operatorname{Spec}(G)=\left\{\lambda_{1}{ }^{m\left(\lambda_{1}\right)}, \lambda_{2}{ }^{m\left(\lambda_{2}\right)}, \ldots, \lambda_{S}{ }^{m\left(\lambda_{S}\right)}\right\}
$$

\section{Circulant matrices}

Fix a positive integer $\mathrm{n} \geq 2$ and let $\mathrm{v}=\left(\mathrm{v}_{0}, \mathrm{v}_{1}, \ldots, \mathrm{v}_{\mathrm{n}-1}\right)$ be a row vector in $\mathbb{C}_{\mathrm{n}}$. Define the shift operator $\mathrm{T}: \mathbb{C}_{\mathrm{n}} \rightarrow \mathbb{C}_{\mathrm{n}}$ by

$$
\mathrm{T}\left(\mathrm{v}_{0}, \mathrm{v}_{1}, \ldots, \mathrm{v}_{\mathrm{n}-1}\right)=\left(\mathrm{v}_{\mathrm{n}-1}, \mathrm{v}_{0}, \ldots, \mathrm{v}_{\mathrm{n}-2}\right) \text {. }
$$

The circulant matrix associated to $\mathrm{v}$ is the $\mathrm{n} \times \mathrm{n}$ matrix whoserows are given by iteration of the shift operator acting on $\mathrm{v}$, that is to say the $\mathrm{k}$-throw is given by $\mathrm{T}^{\mathrm{k}-1} \mathrm{v}, \mathrm{k}=1, \ldots, \mathrm{n}$. Such a matrix will be denoted by $\mathrm{V}=\operatorname{circ}\{\mathrm{v}\}=\operatorname{circ}\left\{\mathrm{v}_{0}, \mathrm{v}_{1}, \ldots, \mathrm{v}_{\mathrm{n}-1}\right\}$.

The following theorem shows how one can calculate eigenvalues and eigenvectors of $\mathrm{V}$.

Theorem 2.1:[8] (Eigenvalues of circulant matrix) Let $v=\left(v_{0}, v_{1}, \ldots, v_{n-1}\right)$ be a row vector in $\mathbb{C}_{n}$, and $V=\operatorname{circ}\{v\}$. If $\varepsilon$ is primitive $n-t h$ root of unity, then

$$
\begin{aligned}
\operatorname{det} V & =\operatorname{det}\left[\begin{array}{ccccc}
v_{0} & v_{1} & \ldots & v_{n-2} & v_{n-1} \\
v_{n-1} & v_{0} & \ldots & v_{n-3} & v_{n-2} \\
\vdots & \vdots & \ddots & \vdots & \vdots \\
v_{2} & v_{3} & \ldots & v_{0} & v_{1} \\
v_{1} & v_{2} & \ldots & v_{n-1} & v_{0}
\end{array}\right] \\
& =\prod_{\ell=0}^{\mathrm{n}-1}\left(\sum_{\mathrm{j}=0}^{\mathrm{n}-1} \varepsilon^{\ell \mathrm{j}} \mathrm{v}_{\mathrm{j}}\right) .
\end{aligned}
$$

\section{Corollary 2.2 [8]}

Eigenvalues of circulant matrix $\mathrm{V}$ is given by the formulae

$$
\lambda_{\ell}=\sum_{\mathrm{j}=0}^{\mathrm{n}-1} \varepsilon^{\ell \mathrm{j}} \mathrm{v}_{\mathrm{j}}, \ell=0, \ldots, \mathrm{n}-1 .
$$

In the coming sections, we will need to use the Chebyshev polynomial of the second kind which is defined by the formula

$$
\mathrm{U}_{\mathrm{n}}(\mathrm{x})=\sin \left(\mathrm{n} \cos ^{-1} \mathrm{x}\right)=\frac{\sin \left((\mathrm{n}+1) \cos ^{-1} \mathrm{x}\right)}{\sqrt{1-\mathrm{x}^{2}}}
$$

And $U_{n}(x)$ satisfies the recursive relation

$$
\mathrm{U}_{0}(\mathrm{x})=1, \mathrm{U}_{1}(\mathrm{x})=2 \mathrm{x},
$$

$\mathrm{U}_{\mathrm{n}}(\mathrm{x})=2 \mathrm{x} \mathrm{U}_{\mathrm{n}-1}(\mathrm{x})-\mathrm{U}_{\mathrm{n}-2}(\mathrm{x}), \mathrm{n} \geq 2$, where

$$
\mathrm{U}_{\mathrm{n}}(\mathrm{x})=\operatorname{det}\left(\begin{array}{cccccc}
2 x & -1 & 0 & \cdots & 0 & 0 \\
-1 & 2 x & -1 & \cdots & 0 & 0 \\
0 & -1 & 2 x & \cdots & 0 & 0 \\
\vdots & \vdots & \vdots & \ddots & \vdots & \vdots \\
0 & 0 & 0 & \cdots & 2 x & -1 \\
0 & 0 & 0 & \cdots & -1 & 2 x
\end{array}\right)
$$

\section{The spectrum of $C_{n}$}

The adjacency matrix of a cycle graph $C_{n}$ is:

$A\left(C_{n}\right)=\left[\begin{array}{ccccc}0 & 1 & 0 & \cdots & 1 \\ 1 & 0 & 1 & \cdots & 0 \\ 0 & 1 & 0 & \cdots & 0 \\ \vdots & \vdots & \vdots & \ddots & \vdots \\ 1 & 0 & 0 & \cdots & 0\end{array}\right]$, where

$a_{i j}=1$ if $|i-j|=1$ or $(n-1)$ and $a_{i j}=0$ otherwise.

\section{Theorem 3.1:}

1) Adjacency spectrum of cycle graph $C_{n}$ are

$$
\lambda_{k}=2 \cos \left(\frac{2 \pi k}{n}\right), k=\{0,1,2, \cdots, n-1\} .
$$

2) Laplacian spectrum of cycle graph $C_{n}$ are

$$
\lambda_{k}=2-2 \cos \left(\frac{2 \pi k}{n}\right), k=\{0,1,2, \cdots, n-1\}
$$

3) Signless Laplacian spectrum of cycle graph $C_{n}$ are

$$
\lambda_{k}=2+2 \cos \left(\frac{2 \pi k}{n}\right), k=\{0,1,2, \cdots, n-1\} .
$$

4) Normalized Laplacian spectrum of cycle graph $C_{n}$ are

$$
\lambda_{k}=1-\cos \left(\frac{2 \pi k}{n}\right), k=\{0,1,2, \cdots, n-1\} .
$$

5) Seidel adjacency spectrum of cycle graph $C_{n}$ are

$$
\lambda_{k}=-2 \cos \left(\frac{2 \pi k}{n}\right)+\frac{\cos (\pi k) \sin \left(\frac{(n-3) \pi k}{n}\right)}{\sin \left(\frac{\pi k}{n}\right)}
$$

$k=\{1,2, \cdots, n-1\}$, and $\lambda_{0}=v_{0}+v_{1}+\cdots+v_{n-1}$, at $k=0$

Proof

1) The adjacency matrix $A\left(C_{n}\right)$ is the circulant matrix with entities

$$
v_{0}=0, v_{1}=1, v_{2}=\ldots=v_{n-2}=0, v_{n-1}=1 \text {. }
$$

Then by properties of circulant matrices its eigenvalues are

$$
\lambda_{k}=v_{0}+v_{1} \varepsilon^{k}+\cdots+v_{n-1} \varepsilon^{(n-1) k}, k=0,1,2, \cdots, n-1,
$$

Where $\varepsilon=e^{\frac{2 \pi i}{n}}$ is the $\mathrm{n}$-th primitive root of the unity. Hence,

$$
\begin{aligned}
\lambda_{k} & =\varepsilon^{k}+\varepsilon^{(n-1) k} \\
& =e^{\frac{2 \pi i}{n} k}+e^{\frac{2 \pi i}{n}(n-1) k} \\
& =e^{\frac{2 \pi i}{n} k}+e^{-\frac{2 \pi i}{n} k}
\end{aligned}
$$




$$
=2 \cos \left(\frac{2 \pi k}{n}\right)
$$

Since $e^{2 \pi i}=\cos 2 \pi+i \sin 2 \pi=1$ Then,

Adjacency spectrum of $C_{n}$ are

$$
\lambda_{k}=2 \cos \left(\frac{2 \pi k}{n}\right), k=\{0,1,2, \cdots, n-1\} .
$$

2) The Laplacian matrix $L\left(C_{n}\right)$ is the circulant matrix with entities

$v_{0}=2, v_{1}=-1, v_{2}=\ldots=v_{n-2}=0, v_{n-1}=-1$

Hence,

$$
\begin{aligned}
\lambda_{k} & =2-\varepsilon^{k}-\varepsilon^{(n-1) k} \\
& =2-e^{\frac{2 \pi i}{n} k}-e^{\frac{2 \pi i}{n}(n-1) k} \\
& =2-2 \cos \left(\frac{2 \pi k}{n}\right)
\end{aligned}
$$

Then, Laplacian spectrum of $C_{n}$ are

$$
\lambda_{k}=2-2 \cos \left(\frac{2 \pi k}{n}\right), k=\{0,1,2, \cdots, n-1\}
$$

3) The Signless Laplacian matrix $Q\left(C_{n}\right)$ is the circulant matrix with entities

$$
v_{0}=2, v_{1}=1, v_{2}=\ldots=v_{n-2}=0, v_{n-1}=1 \text {. }
$$

Hence,

$$
\begin{aligned}
\lambda_{k} & =2+\varepsilon^{k}+\varepsilon^{(n-1) k} \\
& =2+e^{\frac{2 \pi i}{n} k}+e^{\frac{2 \pi i}{n}(n-1) k} \\
& =2+2 \cos \left(\frac{2 \pi k}{n}\right)
\end{aligned}
$$

Then, Signless Laplacian spectrum of $C_{n}$ are

$$
\lambda_{k}=2+2 \cos \left(\frac{2 \pi k}{n}\right), k=\{0,1,2, \cdots, n-1\}
$$

4) The Normalized Laplacian matrix $£\left(C_{n}\right)$ is the circulant matrix with entities

$$
v_{0}=1, v_{1}=-\frac{1}{2}, v_{2}=\ldots=v_{n-2}=0, v_{n-1}=-\frac{1}{2} \text {. }
$$

Hence,

$$
\begin{aligned}
\lambda_{k} & =1-\frac{1}{2} \varepsilon^{k}-\frac{1}{2} \varepsilon^{(n-1) k} \\
& =1-\frac{1}{2} e^{\frac{2 \pi i}{n} k}-\frac{1}{2} e^{\frac{2 \pi i}{n}(n-1) k} \\
& =1-\cos \left(\frac{2 \pi k}{n}\right)
\end{aligned}
$$

Then, Normalized Laplacian spectrum of $C_{n}$ are

$$
\lambda_{k}=1-\cos \left(\frac{2 \pi k}{n}\right), k=\{0,1,2, \cdots, n-1\}
$$

5) The Seidel adjacency matrix $S\left(C_{n}\right)$ is the circulant matrix with entities

$$
v_{0}=0, v_{1}=-1, v_{2}=\ldots=v_{n-2}=1, v_{n-1}=-1 \text {. }
$$

Hence,

$$
\begin{aligned}
\lambda_{k} & =-\varepsilon^{k}+\varepsilon^{2 k}+\varepsilon^{3 k} \cdots+\varepsilon^{(n-2) k}-\varepsilon^{(n-1) k} \\
& =-\left(e^{\frac{2 \pi i}{n} k}+e^{\frac{2 \pi i}{n}(n-1) k}\right)+\left[\varepsilon^{2 k}+\varepsilon^{3 k} \cdots+\varepsilon^{(n-2) k}\right] \\
& =-2 \cos \left(\frac{2 \pi k}{n}\right)+\frac{\varepsilon^{2 k}\left(\varepsilon^{(n-3) k}-1\right)}{\varepsilon^{k}-1} \\
& =-2 \cos \left(\frac{2 \pi k}{n}\right)+\frac{\varepsilon^{2 k} \varepsilon^{\frac{(n-3) k}{2}}\left(\varepsilon^{\frac{(n-3) k}{2}}-\varepsilon^{-\frac{(n-3) k}{2}}\right)}{\varepsilon^{\frac{k}{2}}\left(\varepsilon^{\frac{k}{2}}-\varepsilon^{-\frac{k}{2}}\right)} \\
& =-2 \cos \left(\frac{2 \pi k}{n}\right)+\frac{\varepsilon^{\frac{n k}{2}\left(\varepsilon^{\frac{(n-3) k}{2}}-\varepsilon^{-\frac{(n-3) k}{2}}\right)}}{\varepsilon^{\frac{k}{2}}-\varepsilon^{-\frac{k}{2}}} \\
& =-2 \cos \left(\frac{2 \pi k}{n}\right)+\frac{e^{\pi i k}\left(\varepsilon^{\frac{(n-3) \pi i k}{n}}-\varepsilon^{-\frac{(n-3) \pi i k}{n}}\right)}{\varepsilon^{\frac{\pi i k}{n}}-\varepsilon^{-\frac{\pi i k}{n}}} \\
& =-2 \cos \left(\frac{2 \pi k}{n}\right)+\frac{e^{\pi i k} \sin \left(\frac{(n-3) \pi k}{n}\right)}{\sin \left(\frac{\pi k}{n}\right)} \\
& =-2 \cos \left(\frac{2 \pi k}{n}\right)+\frac{\cos (\pi k) \sin \left(\frac{(n-3) \pi k}{n}\right)}{\sin \left(\frac{\pi k}{n}\right)}
\end{aligned}
$$

Since, $\sin \Theta \theta=\frac{e^{i \theta \theta}-e^{-i \theta \Theta}}{2 i}$ and

$$
e^{\pi i k}=\cos (\pi k)+i \sin (\pi k)=\cos (\pi k)
$$

Then, Seidel adjacency spectrum of $C_{n}$ are

$\lambda_{k}=-2 \cos \left(\frac{2 \pi k}{n}\right)+\frac{\cos (\pi k) \sin \left(\frac{(n-3) \pi k}{n}\right)}{\sin \left(\frac{\pi k}{n}\right)}, k=\{1,2, \cdots, n-1\}$

We have, $\lambda_{0}=v_{0}+v_{1}+\cdots+v_{n-1}$, atk $=0$

\section{On the characteristic polynomials of $P_{n}$}

Our goal is to find a recurrence relationship between the characteristic polynomials of path graphs.

The adjacency matrix of a path $\boldsymbol{P}_{\boldsymbol{n}}$ is:

$A\left(P_{n}\right)=\left[\begin{array}{ccccc}0 & 1 & 0 & \cdots & 0 \\ 1 & 0 & 1 & \cdots & 0 \\ 0 & 1 & 0 & \cdots & 0 \\ \vdots & \vdots & \vdots & \ddots & \vdots \\ 0 & 0 & 0 & \cdots & 0\end{array}\right]$, where

$a_{i j}=1$ if $|i-j|=1$ and $a_{i j}=0$ otherwise.

$$
\varphi\left(P_{n}, \lambda\right)=\operatorname{det}\left(\lambda I-A\left(P_{n}\right)\right)=\operatorname{det}\left[\begin{array}{ccccc}
\lambda & -1 & 0 & \cdots & 0 \\
-1 & \lambda & -1 & \cdots & 0 \\
0 & -1 & \lambda & \cdots & 0 \\
\vdots & \vdots & \vdots & \ddots & \vdots \\
0 & 0 & 0 & \cdots & \lambda
\end{array}\right]
$$

Using cofactor expansion along the first row we get:

$$
\varphi\left(P_{n}, \lambda\right)=\operatorname{det}\left(\lambda I-A_{P_{n}}\right)=\lambda C_{11}+(-1)(-1) C_{12}
$$

We see that $C_{11}$ is the determinant of the matrix $A_{P_{n-1}}$ and $C_{12}$ is the determinant of the matrix:

$$
C_{12}=\operatorname{det}\left[\begin{array}{cccc}
-1 & -1 & \cdots & 0 \\
0 & \lambda & \cdots & 0 \\
\vdots & \vdots & \ddots & \vdots \\
0 & 0 & \cdots & \lambda
\end{array}\right]
$$


Using cofactor expansion along the first column we get that: $C_{12}=-1 C_{11}$

We see that $C_{11}$ is the determinant of the matrix $A_{P_{n-2}}$.

Therefore:

$$
\begin{aligned}
\varphi\left(P_{n}, \lambda\right) & =\operatorname{det}\left(\lambda I-A_{P_{n}}\right) \\
& =\lambda \operatorname{det}\left(\lambda I-A_{P_{n-1}}\right)-\operatorname{det}\left(\lambda I-A_{P_{n-2}}\right) \\
& =\lambda \varphi\left(P_{n-1}, \lambda\right)-\varphi\left(P_{n-2}, \lambda\right) \forall n \geq 3
\end{aligned}
$$

Note that this implies $\varphi\left(P_{n}, \lambda\right)=U_{n}\left(\frac{\lambda}{2}\right)$ where

$$
U_{n}(x)=\sin \left(n \cos ^{-1} x\right)=\frac{\sin \left((n+1) \cos ^{-1} x\right)}{\sqrt{1-x^{2}}}
$$

Thus $\varphi\left(P_{n}, \lambda\right)=U_{n}\left(\frac{\lambda}{2}\right)=\frac{\sin \left((n+1) \cos ^{-1} \frac{\lambda}{2}\right)}{\sqrt{1-\left(\frac{\lambda}{2}\right)^{2}}}$,

Let $\cos ^{-1} \frac{\lambda}{2}=\theta \Rightarrow \frac{\lambda}{2}=\cos \theta$

Then

$$
\varphi\left(P_{n}, \lambda\right)=\frac{\sin ((n+1) \theta)}{\sin \theta}
$$

If $\varphi\left(P_{n}, \lambda\right)=0$ we get

$$
\sin ((n+1) \theta)=0 \text { and } \sin \theta \neq 0 \Rightarrow \theta \neq \pi k
$$

Thus $(n+1) \theta=\pi k \Rightarrow \theta=\frac{\pi k}{n+1} \Rightarrow \cos \theta=\cos \left(\frac{\pi k}{n+1}\right)$,

$$
\lambda_{k}=2 \cos \left(\frac{\pi k}{n+1}\right), k=\{1,2, \cdots, n\} \text {. }
$$

Adjacency Spectrum of $P_{n}$ are $\lambda_{\mathrm{k}}=2 \cos \left(\frac{\pi \mathrm{k}}{\mathrm{n}+1}\right), \mathrm{k}=\{1,2, \cdots, \mathrm{n}\}$.

Laplacian Matrix of $\boldsymbol{P}_{\boldsymbol{n}}$ : The Laplacian matrix of $P_{n}$ has the form

$$
L\left(P_{n}\right)=\left[\begin{array}{ccccc}
1 & -1 & 0 & \cdots & 0 \\
-1 & 2 & -1 & \cdots & 0 \\
0 & -1 & 2 & \cdots & 0 \\
\vdots & \vdots & \vdots & \ddots & \vdots \\
0 & 0 & 0 & \cdots & 1
\end{array}\right]
$$

$$
p\left(P_{n}, \rho\right)=\operatorname{det}\left(L\left(P_{n}\right)-\rho I\right)
$$

$$
\begin{gathered}
=\operatorname{det}\left(\begin{array}{cccccc}
1-\rho & -1 & 0 & \cdots & 0 & 0 \\
-1 & 2-\rho & -1 & \cdots & 0 & 0 \\
0 & -1 & 2-\rho & \cdots & 0 & 0 \\
\vdots & \vdots & \vdots & \ddots & \vdots & \vdots \\
0 & 0 & 0 & \cdots & 2-\rho & -1 \\
0 & 0 & 0 & \cdots & -1 & 1-\rho
\end{array}\right) \\
=\operatorname{det}\left(\begin{array}{cccccc}
2-\rho & -1 & 0 & \cdots & 0 & 0 \\
-1 & 2-\rho & -1 & \cdots & 0 & 0 \\
0 & -1 & 2-\rho & \cdots & 0 & 0 \\
\vdots & \vdots & \vdots & \ddots & \vdots & \vdots \\
0 & 0 & 0 & \cdots & 2-\rho & -1 \\
0 & 0 & 0 & \cdots & -1 & 1-\rho
\end{array}\right) \\
-\operatorname{det}\left(\begin{array}{ccccccc}
1 & 0 & 0 & \cdots & 0 & 0 \\
-1 & 2-\rho & -1 & \cdots & 0 & 0 \\
0 & -1 & 2-\rho & \cdots & 0 & 0 \\
\vdots & \vdots & \vdots & \ddots & \vdots & \vdots \\
0 & 0 & 0 & \cdots & 2-\rho & -1 \\
0 & 0 & 0 & \cdots & -1 & 1-\rho
\end{array}\right)
\end{gathered}
$$

$$
\begin{gathered}
=\operatorname{det}\left(\begin{array}{cccccc}
2-\rho & -1 & 0 & \cdots & 0 & 0 \\
-1 & 2-\rho & -1 & \cdots & 0 & 0 \\
0 & -1 & 2-\rho & \cdots & 0 & 0 \\
\vdots & \vdots & \vdots & \ddots & \vdots & \vdots \\
0 & 0 & 0 & \cdots & 2-\rho & -1 \\
0 & 0 & 0 & \cdots & -1 & 1-\rho
\end{array}\right) \\
-\operatorname{det}\left(\begin{array}{cccccc}
2-\rho & -1 & 0 & \cdots & 0 & 0 \\
-1 & 2-\rho & -1 & \cdots & 0 & 0 \\
0 & -1 & 2-\rho & \cdots & 0 & 0 \\
\vdots & \vdots & \vdots & \ddots & \vdots & \vdots \\
0 & 0 & 0 & \cdots & 2-\rho & -1 \\
0 & 0 & 0 & \cdots & -1 & 1-\rho
\end{array}\right)
\end{gathered}
$$$$
=V_{n}-V_{n-1} \text {, where }
$$

$$
V_{n}=\operatorname{det}\left(\begin{array}{cccccc}
2-\rho & -1 & 0 & \cdots & 0 & 0 \\
-1 & 2-\rho & -1 & \cdots & 0 & 0 \\
0 & -1 & 2-\rho & \cdots & 0 & 0 \\
\vdots & \vdots & \vdots & \ddots & \vdots & \vdots \\
0 & 0 & 0 & \cdots & 2-\rho & -1 \\
0 & 0 & 0 & \cdots & -1 & 1-\rho
\end{array}\right)
$$

$$
=\operatorname{det}\left(\begin{array}{cccccc}
2-\rho & -1 & 0 & \cdots & 0 & 0 \\
-1 & 2-\rho & -1 & \cdots & 0 & 0 \\
0 & -1 & 2-\rho & \cdots & 0 & 0 \\
\vdots & \vdots & \vdots & \ddots & \vdots & \vdots \\
0 & 0 & 0 & \cdots & 2-\rho & -1 \\
0 & 0 & 0 & \cdots & -1 & 2-\rho
\end{array}\right)
$$

$$
\begin{aligned}
& -\operatorname{det}\left(\begin{array}{cccccc}
2-\rho & -1 & 0 & \cdots & 0 & 0 \\
-1 & 2-\rho & -1 & \cdots & 0 & 0 \\
0 & -1 & 2-\rho & \cdots & 0 & 0 \\
\vdots & \vdots & \vdots & \ddots & \vdots & \vdots \\
0 & 0 & 0 & \cdots & 2-\rho & -1 \\
0 & 0 & 0 & \cdots & 0 & 1
\end{array}\right) \\
= & U_{n}\left(\frac{2-\rho}{2}\right)-U_{n-1}\left(\frac{2-\rho}{2}\right) .
\end{aligned}
$$

Here $U_{n}(x)$ is a Chebyshev polynomial of the second kind. Since $U_{n}(x)-2 x U_{n-1}(x)+U_{n-2}(x)=0$, we obtain

$$
\begin{aligned}
& p\left(P_{n}, \rho\right)=\operatorname{det}\left(L\left(P_{n}\right)-\rho I\right)=V_{n}-V_{n-1} \\
& =U_{n}\left(\frac{2-\rho}{2}\right)-U_{n-1}\left(\frac{2-\rho}{2}\right)-U_{n-1}\left(\frac{2-\rho}{2}\right)+U_{n-2}\left(\frac{2-\rho}{2}\right) \\
& =U_{n}\left(\frac{2-\rho}{2}\right)+U_{n-2}\left(\frac{2-\rho}{2}\right)-2 U_{n-1}\left(\frac{2-\rho}{2}\right) \\
& =2\left(\frac{2-\rho}{2}\right) U_{n-1}\left(\frac{2-\rho}{2}\right)-2 U_{n-1}\left(\frac{2-\rho}{2}\right) \\
& =-\rho U_{n-1}\left(\frac{2-\rho}{2}\right) .
\end{aligned}
$$

Then, the Laplacian Spectrum of $P_{n}$ are $\rho_{k}=2-2 \cos \left(\frac{\pi k}{n}\right), k=\{0,1,2, \cdots, n-1\}$.

SignlessLaplacian Matrix of $\boldsymbol{P}_{\boldsymbol{n}}$ : The SignlessLaplacian matrix of $P_{n}$ is the matrix $Q\left(P_{n}\right)=D+A_{P_{n}}$, 


$$
Q\left(P_{n}\right)=\left[\begin{array}{ccccc}
1 & 1 & 0 & \cdots & 0 \\
1 & 2 & 1 & \cdots & 0 \\
0 & 1 & 2 & \cdots & 0 \\
\vdots & \vdots & \vdots & \ddots & \vdots \\
0 & 0 & 0 & \cdots & 1
\end{array}\right]
$$

$F\left(P_{n}, \mu\right)=\operatorname{det}\left(\mu I-L_{P_{n}}\right)$

$$
=\operatorname{det}\left(\begin{array}{cccccc}
\mu-1 & -1 & 0 & \cdots & 0 & 0 \\
-1 & \mu-2 & -1 & \cdots & 0 & 0 \\
0 & -1 & \mu-2 & \cdots & 0 & 0 \\
\vdots & \vdots & \vdots & \ddots & \vdots & \vdots \\
0 & 0 & 0 & \cdots & \mu-2 & -1 \\
0 & 0 & 0 & \cdots & -1 & \mu-1
\end{array}\right)
$$$$
=\operatorname{det}\left(\begin{array}{cccccc}
\mu-2 & -1 & 0 & \cdots & 0 & 0 \\
-1 & \mu-2 & -1 & \cdots & 0 & 0 \\
0 & -1 & \mu-2 & \cdots & 0 & 0 \\
\vdots & \vdots & \vdots & \ddots & \vdots & \vdots \\
0 & 0 & 0 & \cdots & \mu-2 & -1 \\
0 & 0 & 0 & \cdots & -1 & \mu-1
\end{array}\right)
$$$$
+\operatorname{det}\left(\begin{array}{cccccc}
1 & 0 & 0 & \cdots & 0 & 0 \\
-1 & \mu-2 & -1 & \cdots & 0 & 0 \\
0 & -1 & \mu-2 & \cdots & 0 & 0 \\
\vdots & \vdots & \vdots & \ddots & \vdots & \vdots \\
0 & 0 & 0 & \cdots & \mu-2 & -1 \\
0 & 0 & 0 & \cdots & -1 & \mu-1
\end{array}\right)
$$$$
=\operatorname{det}\left(\begin{array}{cccccc}
\mu-2 & -1 & 0 & \cdots & 0 & 0 \\
-1 & \mu-2 & -1 & \cdots & 0 & 0 \\
0 & -1 & \mu-2 & \cdots & 0 & 0 \\
\vdots & \vdots & \vdots & \ddots & \vdots & \vdots \\
0 & 0 & 0 & \cdots & \mu-2 & -1 \\
0 & 0 & 0 & \cdots & -1 & \mu-1
\end{array}\right)
$$$$
+\operatorname{det}\left(\begin{array}{cccccc}
\mu-2 & -1 & 0 & \cdots & 0 & 0 \\
-1 & \mu-2 & -1 & \cdots & 0 & 0 \\
0 & -1 & \mu-2 & \cdots & 0 & 0 \\
\vdots & \vdots & \vdots & \ddots & \vdots & \vdots \\
0 & 0 & 0 & \cdots & \mu-2 & -1 \\
0 & 0 & 0 & \cdots & -1 & \mu-1
\end{array}\right)
$$

$=V_{n}+V_{n-1}$, where

$$
\begin{array}{r}
V_{n}=\operatorname{det}\left(\begin{array}{cccccc}
\mu-2 & -1 & 0 & \cdots & 0 & 0 \\
-1 & \mu-2 & -1 & \cdots & 0 & 0 \\
0 & -1 & \mu-2 & \cdots & 0 & 0 \\
\vdots & \vdots & \vdots & \ddots & \vdots & \vdots \\
0 & 0 & 0 & \cdots & \mu-2 & -1 \\
0 & 0 & 0 & \cdots & -1 & \mu-1
\end{array}\right) \\
=\operatorname{det}\left(\begin{array}{cccccc}
\mu-2 & -1 & 0 & \cdots & 0 & 0 \\
-1 & \mu-2 & -1 & \cdots & 0 & 0 \\
0 & -1 & \mu-2 & \cdots & 0 & 0 \\
\vdots & \vdots & \vdots & \ddots & \vdots & \vdots \\
0 & 0 & 0 & \cdots & \mu-2 & -1 \\
0 & 0 & 0 & \cdots & -1 & \mu-2
\end{array}\right)
\end{array}
$$$$
+\operatorname{det}\left(\begin{array}{cccccc}
\mu-2 & -1 & 0 & \cdots & 0 & 0 \\
-1 & \mu-2 & -1 & \cdots & 0 & 0 \\
0 & -1 & \mu-2 & \cdots & 0 & 0 \\
\vdots & \vdots & \vdots & \ddots & \vdots & \vdots \\
0 & 0 & 0 & \cdots & \mu-2 & -1 \\
0 & 0 & 0 & \cdots & 0 & 1
\end{array}\right)
$$$$
=U_{n}\left(\frac{\mu-2}{2}\right)+U_{n-1}\left(\frac{\mu-2}{2}\right) .
$$

Thus $F\left(P_{n}, \mu\right)=V_{n}+V_{n-1}=\mu U_{n-1}\left(\frac{\mu-2}{2}\right)$

Thus, Signless Laplacian Spectrum of $P_{n}$ are $\mu_{n}=2+2 \cos \frac{\pi k}{n}, k=\{1,2, \cdots, n\} \forall n \geq 3$

Theorem Kel'mans [14], Let $G+H$ denote the join of $G$ and $H$, then

$$
P(G+H, \lambda)=\frac{\lambda\left(\lambda-n_{1}-n_{2}\right)}{\left(\lambda-n_{1}\right)\left(\lambda-n_{2}\right)} P\left(G, \lambda-n_{2}\right) P\left(H, \lambda-n_{1}\right)
$$

Where $n_{1}$ and $n_{2}$ are orders of $G$ and $H$, respectively $P(G, \lambda)$ is the characteristic polynomial of the Laplacian matrix of $G$.

- The wheel graph, $W_{n+1}$ the graph which is given by $W_{n+1}=K_{1}+C_{n}$, where $C_{n}$ is the cycle graph with n vertices and $K_{1}$ is any new vertex.

- The fan graph, $\mathrm{F}_{\mathrm{n}+1}$ the graph which is given by $F_{n+1}=K_{1}+P_{n}$, where $P_{n}$ is the path graph with $n$ vertices and $K_{1}$ is any new vertex.
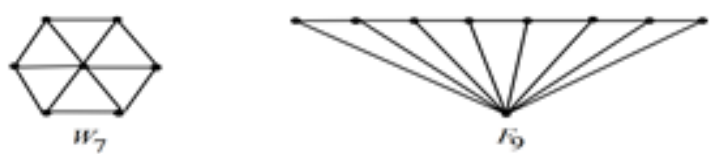

Fig. 1

Theorem 4.1:

1) Laplacian spectrum of the fan graph $F_{n+1}$ are $\left\{0, n+1,3-2 \cos \frac{\pi k}{n}, k=1, \ldots, n-1\right\}$

2) Laplacian spectrum of the wheel graph $W_{n+1}$ are Proof $\left\{0, \mathrm{n}+1,3-2 \cos \frac{2 \pi \mathrm{k}}{\mathrm{n}}, \mathrm{k}=1, \ldots, \mathrm{n}-1\right\}$.

1) Since Laplacian polynomial of the path graph $P\left(P_{n}, \lambda\right)=-\lambda U_{n-1}\left(\frac{2-\lambda}{2}\right)$ and $P\left(K_{1}, \lambda\right)=\lambda$, then by theorem (Kel'mans) the Laplacian polynomial of the fan graph $F_{n+1}$ is givenby the formula $P\left(F_{n+1}, \lambda\right)=-\lambda(\lambda-n-1) U_{n-1}\left(\frac{3-\lambda}{2}\right)$.

Thus Laplacian spectrum of $F_{n+1}$ are

$$
\left\{0, n+1,3-2 \cos \frac{\pi k}{n}, k=1, \ldots, n-1\right\}
$$

2) Since Laplacian polynomial of the cycle graph $\mathrm{P}\left(\mathrm{C}_{\mathrm{n}}, \lambda\right)=2 \lambda\left[\mathrm{T}_{\mathrm{n}-1}\left(\frac{2-\lambda}{2}\right)-1\right]$ and $\mathrm{P}\left(\mathrm{K}_{1}, \lambda\right)=\lambda$, then by theorem (Kel'mans) the Laplacian polynomial of the wheel graph $\mathrm{W}_{\mathrm{n}+1}$ is givenby the formula

$$
\begin{gathered}
\mathrm{P}\left(\mathrm{W}_{\mathrm{n}+1}, \lambda\right)=2 \lambda(\lambda-\mathrm{n}-1)\left[\mathrm{T}_{\mathrm{n}-1}\left(\frac{3-\lambda}{2}\right)-1\right] \quad \text { we get } \\
\mathrm{T}_{\mathrm{n}-1}\left(\frac{3-\lambda}{2}\right)=\cos \left((\mathrm{n}-1) \cos ^{-1} \frac{3-\lambda}{2}\right), \text { let } \\
\cos ^{-1} \frac{3-\lambda}{2}=\theta \Rightarrow \frac{3-\lambda}{2}=\cos \theta \text {, thus } \\
\mathrm{T}_{\mathrm{n}-1}\left(\frac{3-\lambda}{2}\right)=\cos ((\mathrm{n}-1) \theta), \text { if } \\
T_{n-1}\left(\frac{3-\lambda}{2}\right)=1 \Rightarrow \cos ((n-1) \theta)=1 \\
\Rightarrow(n-1) \theta=2 \pi k \Rightarrow \theta=\frac{2 \pi k}{n-1} \Rightarrow \cos \theta=\cos \left(\frac{2 \pi k}{n-1}\right), \\
k=\{0,1,2, \cdots, n-2\}
\end{gathered}
$$

Where $\cos \theta=\frac{3-\lambda}{2}$, then $\lambda=3-2 \cos \frac{2 \pi k}{n}, k=1, \ldots, n-1$.

Thus Laplacian spectrum of $W_{n+1}$ are 
$\left\{0, n+1,3-2 \cos \frac{2 \pi \mathrm{k}}{\mathrm{n}}, \mathrm{k}=1, \ldots, \mathrm{n}-1\right\}$

\section{References}

[1] Ayoobi,F., Omidi,G. R. and Tayfeh-Rezaie,B. "A note on graphs whosesignlessLaplacian has three distinct eigenvalues", Lin. Multilin. Alg. 59(2011) 701-706. (p. 220)

[2] Biggs, N.L. "Algebraic Graph Theory", Cambridge University Press, 2nd edition,Cambridge, 1993.

[3] Bondarenko, A. V. and Radchenko,D. V. "On a family of strongly regulargraphs with $\lambda=1$ ”, arXiv:1201.0383, Feb. 2012. (p. 132).

[4] Boulet, R."Disjoint unions of complete graphs characterized by their Laplacian spectrum", Electr. J. Lin. Alg. 18 (2009) 773-783. (p. 204).

[5] Cvetković, D., Doob, M. and H. Sachs, H. "Spectra of Graphs, Theory andApplications", Academic Press, 1980.

[6] Cvetkovic, D., Rowlinson, P., and Simic, S. (2010) "An Introduction to the Theory of Graph Spectra Cambridge U.P.", New York.

[7] Das, K.C. (2004) "The Laplacian spectrum of a graph",Comput Math.Appl.48,715724.http://dx.doi.org/10.1016/j.camwa.2004.05.0 $\underline{05}$.

[8] Davis,P.J. "Circulant Matrices", AMS Chelsea Publishing, 1994.

[9] El seedy, E., Hussein, S. and AboElkher, A. "Spectra ofsome simple graphs",Math. Theory and Mod. 5 (2015) 115-121.

[10] Fiedler, M. "Algebraic connectivity of graphs", Czech. Math. J., $1973 ; 23: 298-305$.

[11] Godsil C. and Royle, G. "Algebraic Graph Theory”, Springer Verlag, New York, 2001. http://dx.doi.org/10.1007/978-1-4613-0163-9.

[12] Kaveh, A. "Structural Mechanics: Graph and Matrix Methods", 3rd edition, ResearchStudies Press, Somerset, UK, 2004.

[13] Kaveh, A. "Optimal Structural Analysis", 2nd edition, John Wiley, UK, 2006.http://dx.doi.org/10.1002/9780470033326.

[14] Kel'mans,A.K. "The number of trees in a graph". I. Automat iTelemeh. 26 (1965)2194-2204 (in Russian); transl. Automat. Remote Control 26 (1965) 2118-2129.

[15] Mohar, B. "The Laplacian spectrum of graphs, entitled Graph Theory, Combinatorics and Applications", edit. Y. Alavi et al., Vol. 2, John Wiley, NY, 1991, pp 871-898.

[16] Pothen, A. Simon, H. and Liou, K.P. "Partitioning sparse matrices with eigenvectors of graphs", SIAM J. Matrix Anal. Appl., 1990; 11:430-452. http://dx.doi.org/10.1137/0611030.

[17] Spielman, D. (2004) "Spectral graph theory and its Applications", lecture notes fall, onlineviahttp://www.cs.yale.edu/homes/spielman/eigs

[18] Topping, BHV and Sziveri, J. "Parallel subdomain generation method”, Proc. CivilComp, Edinburgh, UK, 1995. 